\title{
DISTORSION OF ISOCHROMATIC FRINGES IN THE PROCESS OF STRESS MEASUREMENT IN STRUCTURAL PART BY PHOTOELASTICIMETRY
}

\author{
FRANKOVSKÝ Peter ${ }^{1}$, OSTERTAG Oskar¹, DELYOVÁ Ingrid ${ }^{1}$ \\ ${ }^{1}$ Technical University of Košice, Faculty of Mechanical Engineering, Department of Applied Mechanics \\ and Mechanical Engineering, Letná 9, 04200 Košice, Slovak Republic, email: peter.frankovsky@tuke.sk
}

\begin{abstract}
In the article we show the possibility of distorting the results of the experiment by photoelasticity path, in case the isochromatic fringe patterns are recorded by the camera. The recording of the color spectrum may be distorted by inappropriate selection of the recording medium, which is reflected by different types of aberrations. Transferring a record to a computer allows you to automate the experiment process, thus saving time to work with isochromatic fringes. As an example, we present the experimental determination of the stresses of the rotor with a symmetrical arm. The analysis was performed using software developed by the authors. This software enables full automation of the experiment process. The rotor is investigated by the method of reflection photoelasticity. The experimental solution is realized as a case of periodically repeating dynamic effects, in which a stroboscopic source of white light is needed. The results of the experiment are compared with the numerical solution and their dependence is represented by diagram.
\end{abstract}

KEYWORDS: aberration, photoelasticity, isochromatic and isoclinic fringes

\section{$1 \quad$ Introduction}

From economic point of view, an experimental determination of vulnerable parts of a loaded construction or its bearing elements needs maximum reduction of time. This may be reached by automated process of the experiment with utilization of any available high-quality means. The maximum demanded software combined with advanced experimental equipment enables a variability of analyses along with high-quality and fast interpretation of experimental results with perfect graphical presentation of key details. One of the methods based on the electromagnetic wave combined with technical support along with utilization of a high-quality recording medium may represent a probable solution. Through the chosen method and based on the photosensitive coating isoline image, or the model, it is possible to come to quick conclusions considering the areas of concentrated stresses and to evaluate these areas in an effective way. In order to verify this method and to establish a systematic procedure, the first phase of our solution was focused on the versatile balanced task of twodimensional both isotropic and orthotropic backgrounds. Regarding the stress analysis we decided to use the method of characteristics. The net of specific curves created by this method is most similar to the curves (opposite to well known method of rectangular net) incipient under temporary light refraction by means of photosensitive model or reflection coating. It is necessary to point out that this solution enables the elimination of subjective errors made by the experimenter when identifying the isolines identification [1]. 


\section{Mathematical identification of the order of isochromatic fringes and separation of the principal stresses of the structural element}

Determination of the isochromatic fringe order based on the color spectrum image with consecutive separation of principle stresses is that part of the experiment the results of which are influenced by individual abilities and experience of the experimenter. In an attempt to avoid subjective evaluation of the measurement and thereby the incidental and systematic errors too, we decided to automate the course of the experiment in full extent. The subjective reading of the isochromatic fringe order was replaced by the camcorder, or more precisely digital camera, and the separation of stresses was operated by the „Photoelast“ software developed for this purpose. Regarding the development of the measurement evaluation program it was necessary to determine an appropriate method by what it would be possible to separate the stresses. For this purpose we chose the method of characteristics not demanding any supplementary information gained from the isoclines. The other methods were considered too (the method of sliding stress differences, the method of integration along the trajectories of principal stresses and others [2,3]. Thus, regarding specific methods applicable for the solution of first-order hyperbolic systems we decided to use the method of characteristics which was correct in case of two independent variables and the unknown functions. Especially in case of two equations system with two unknown functions it seems to be one of the most effective numerical methods and it is particularly suitable for programming within the commercial program landscape. The stated mathematical method, carried out by means of additional experimental information, has led us to differential equations of hyperbolic type as derived in the work [4].

$$
\begin{aligned}
& k_{f} \frac{\partial}{\partial x}\left(\frac{\varepsilon_{s}}{f_{\varepsilon}}\right)+\frac{\partial N}{\partial x} \cos 2 \varphi+\frac{\partial N}{\partial y} \sin 2 \varphi-2 N\left(\frac{\partial \varphi}{\partial x} \sin 2 \varphi-\frac{\partial \varphi}{\partial y} \cos 2 \varphi\right)=0, \\
& k_{f} \frac{\partial}{\partial y}\left(\frac{\varepsilon_{s}}{f_{\varepsilon}}\right)+\frac{\partial N}{\partial x} \sin 2 \varphi+\frac{\partial N}{\partial y} \cos 2 \varphi-2 N\left(\frac{\partial \varphi}{\partial y} \sin 2 \varphi-\frac{\partial \varphi}{\partial x} \cos 2 \varphi\right)=0,
\end{aligned}
$$

with index $f=r, t$.

For the reflection photoelasticity we apply the ratio $k_{r}=2 k$ and for the transmission $k_{t}=k$, where $k=(1+\mu) /(1-\mu)$, in which $\mu$ represents Poisson's ratio of the experimental material.

With application to orthotropic landscape we gained the equation patterns as follows:

$$
\begin{aligned}
& 2 k_{1} \frac{\partial}{\partial \xi}\left(\frac{\varepsilon_{s}}{f_{\varepsilon}}\right)+k_{2} \frac{\partial N}{\partial \xi} \cos 2 \varphi+k_{4} \frac{\partial N}{\partial \eta} \sin 2 \varphi-2 N\left(k_{2} \frac{\partial \varphi}{\partial \xi} \sin 2 \varphi-k_{4} \frac{\partial \varphi}{\partial \eta} \cos 2 \varphi\right)=0, \\
& 2 k_{2} \frac{\partial}{\partial \eta}\left(\frac{\varepsilon_{s}}{f_{\varepsilon}}\right)+k_{4} \frac{\partial N}{\partial \xi} \sin 2 \varphi+k_{3} \frac{\partial N}{\partial \eta} \cos 2 \varphi-2 N\left(k_{3} \frac{\partial \varphi}{\partial \eta} \sin 2 \varphi-k_{4} \frac{\partial \varphi}{\partial \xi} \cos 2 \varphi\right)=0,
\end{aligned}
$$

where the constants $k_{1}, k_{2}, k_{3}, k_{4}$ were substituted by $k_{1}=Q_{11}+2 Q_{12}+Q_{22}, k_{2}=Q_{11}-Q_{22}$, $k_{3}=Q_{11}-2 Q_{12}+Q_{22}, \quad k_{4}=2 Q_{66}, \quad$ where $\quad Q_{11}=E_{L} /\left(1-\mu_{L T} \mu_{T L}\right), \quad Q_{22}=E_{T} /\left(1-\mu_{L T} \mu_{T L}\right)$, $Q_{12}=\mu_{L T} E_{T} /\left(1-\mu_{L T} \mu_{T L}\right)=\mu_{T L} E_{L} /\left(1-\mu_{L T} \mu_{T L}\right), Q_{66}=G_{L T}$

the elastic modules were applied in technical practice. Within the plane task the indexes $L, T$ represent both longitudinal and transversal orientations of natural axes of the orthotropy [5].

The system of partial differential equations (1) and (2) represents the quasi-linear equations of hyperbolic type where as unknown are regarded partial derivations of quested functions represented by the expression $\varepsilon_{s} / f_{\varepsilon}$, where $\varepsilon_{s}=\left(\varepsilon_{1}+\varepsilon_{2}\right) / 2$ are the mean principal strains. The angle $\varphi$ represents the larger of the principal strains deviation from generally chosen axes $x, y$, or more precisely, the axes of the orthotropy $\xi, \eta$. The non-dimensional parameter $f_{\varepsilon}$ is the fringe constant of the model strain or the reflection coating and is depending on the rate of sensitivity of the optically sensitive material. The isochromatic fringe order value $N$ found on 
the reflection material coated on the examined surface, or more precisely on the transmission model, may be experimentally determined for ideal elastic material with the stress linear depending on the strains, within the equations (1). The same procedure we apply for the determination of $N$ within the equations (2) and for this case we consider only the reflection coating on the orthotropic material surface. The parameters $\varphi$ and $N$ are depending on the coordinates $x, y$, or more precisely $\xi, \eta$. The presented solution enables us to find the state of stress of the examined element right by the observation of the colour spectrum, thus the isochromatic fringes. In this case it is not necessary to identify the isoclinic fringes as they simplify the process of the experiment.

\section{Aberration impact on results of the experiment}

Automated experiment within the photoelasticity requires recording of isolines by means of a recording technique. The recording technique may include the camcorder, or more precisely digital camera.

However, the photographic way of recording the isolines has its own problems. When using the reflection polariscope the light ray passes the photoactive prism two times and it results in the angle variance at the analyzer unit. By camcorder or by digital camera (compact or digital single-lens reflex camera) documented isolines of temporary loaded photosensitive materials bring the principal problem of various types of aberration. Aberration of optical systems is divided into monochromatic and chromatic ones (Fig. 1).

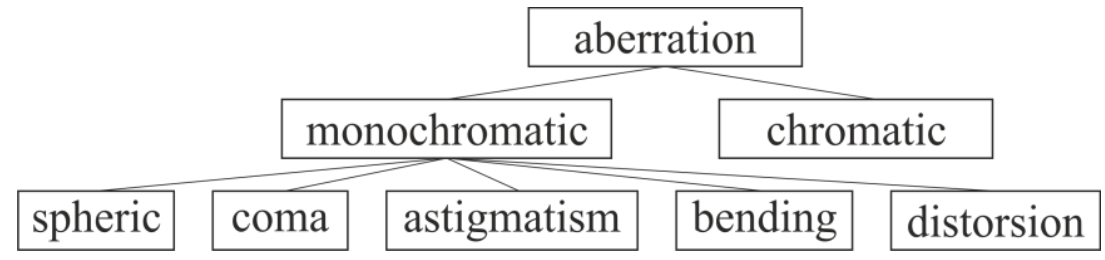

Fig. 1 Optical systems aberration scheme

Monochromatic aberration is defined by loss of the real image. The scanned points of the real image (Fig. 2a), apart from the central, at the spheric aberration seem to be enlarged and in the direction from the centre are blurred (Fig. 2b). At the coma aberration (Fig. 2c) the scanned points in the direction from the centre are blurred to the shape of the small drops with "the core" and "the tail". At the astigmatic aberration (Fig. 2d, e) the surrounding points, apart from the centre, are blurred to the segments ordered to the image tangentially, or more precisely radially.

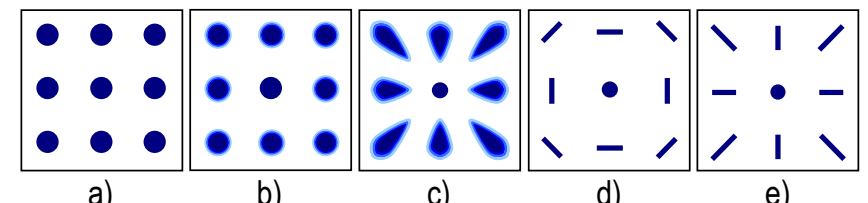

Fig. 2 Aberration example presentation: a) ideal image, b) spheric, c) coma, d) and e) astigmatism

When recording the isolines chromatism is a considerable problem too. The reason of it is the dependency of the refraction index on the light wave length. Following the different wave length of the spectral colors the rays are not intersecting in one focus (the line of focuses is forming). Closest behind the lens is the focus of the violet color tones and farthest is the focus of the red color (Fig. 3). This feature results in the state when the inscription is put blurry on the image sensor. The chromatic aberration may be eliminated by using the digital camera (digital single-lens reflex camera) with replaceable objective. Better marked it is when using the compact digital cameras. 


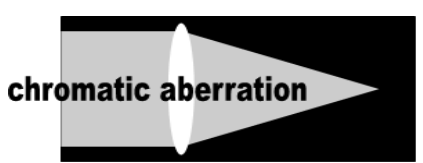

a)

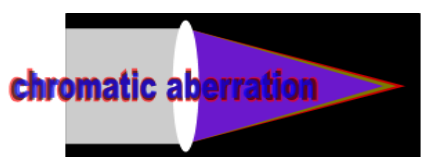

b)

Fig. 3 Chromatic aberration: a) ideal state, b) scanned image

The system of the equations (1) and (2) was the supporting member of the „Photoelast“ program preparation. In addition, the solution was required to be integrated with the program identifying the level of RGB (Red, Green, Blue) components of the isochromatic curves color spectrum moving within the interval of units from 0 to 255 [6]. In the course of the experiment, however, we noticed the problem of aberration. At the automation of the experiment, the aberration implied improperly determined parameter of the color spectrum unit level and, at the same time, errors arising during the determination of construction element stress. In some cases, in the area under examination, the respective isochromatic fringes were evaluated by „Photoelast“ software determining identical number of RGB units, thus the isochromatic fringes showed the same order. Therefore, as the result of several examined alternatives, we decided to use the digital single-lens reflex camera with removable objective and recording in the *.raw format, as the medium transmitting the color spectrum image.

\section{Application of the experiment}

During the first phase we decided the presented program to be verified through the samples symmetric as to both geometry and stress. It was decided about this solution for the purposes of a simple check of the measuring system.

The initial stage of the experimental analysis based on reflection photoelasticity included a general analysis of stress distribution in the analyzed rotors. A picture of isochromatic fringes is a sufficient outcome of such analysis. Within the experiment the photosensitive material PS-1A was applied while its material features are shown in related catalogue, and the reflection polariscope LF/Z-2 Vishay was use.

The rotor was then observed under circular polarized light. The resulting isochromatic fringes (colorful lines or areas) represent connecting lines of points along which the difference of principal normal stresses $\sigma_{1}-\sigma_{2}$ is constant. The picture of isochromatics enables us to visually observe the distribution of principal stresses over the whole surface of the rotor. It is also possible to immediately determine the high-stress areas or stress concentrators [7]. Fig. 4 shows isochromatic fringes on the rotor at 5.000 RPM [6, 8, 9].

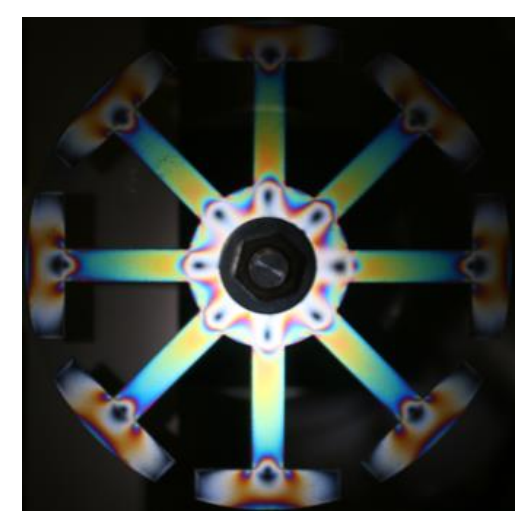

Fig. 4 Isochromatic fringes on the sample rotor 
Based on the observation of isochromatic fringes on the sample rotor with symmetrical arm end (Fig. 4) it can be concluded that during rotation of the rotor a biaxial stress state occurs in the disc as well as the segment and a uniaxial stress state occurs in the arm. Biaxial stress state occurs in intermediate parts of the object when the rotor is rotating. In this case, the arm is loaded by uniaxial tensile loads.

For the comparison of differences of principal normal stress $\sigma_{1}-\sigma_{2}$ and their components $\sigma_{1}, \sigma_{2}$ in the analyzed points obtained by experimental means of reflection photoelasticity the analyzed sample rotors were modeled in SolidWorks program, and stress values were calculated with the use of final element method. Fig. 5 shows a field of reduced stresses on the sample according to Guest hypothesis $[10,11]$.
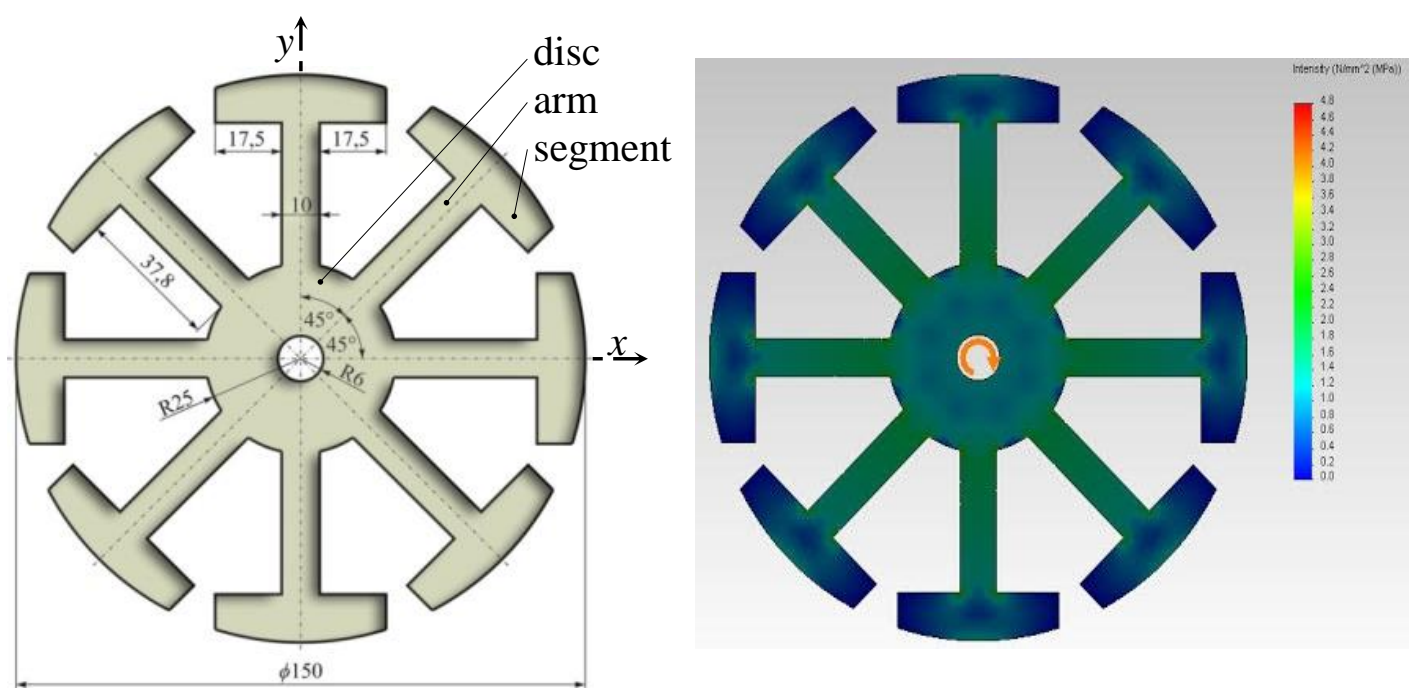

Fig. 5 Isochromatic fringes on the sample rotor

Quantitative determination of the difference of principal normal stresses $\sigma_{\text {red }}=\sigma_{1}-\sigma_{2}$ (Tab. 1) and separated stress values $\sigma_{1}, \sigma_{2}$ (Tab. 1) was done by „Photoelast“" software on the basis of images of isoclinic and isochromatic fringes. Stress values were determined in points with coordinates listed in Tab. 1.

Tab. 1 Values of identified normal stresses $\sigma_{\text {red }}, \sigma_{1}$ and $\sigma_{2}$

\begin{tabular}{|c|c|c|c|c|c|c|}
\hline \multirow{2}{*}{$\begin{array}{c}\text { Coordinates } \\
\text { of examined } \\
\text { points } x_{i} \\
{[\mathrm{~mm}]}\end{array}$} & \multicolumn{2}{|c|}{$\sigma_{\text {red }}[\mathrm{MPa}$ determined: } & \multicolumn{4}{|c|}{$\sigma_{1}, \sigma_{2}[\mathrm{MPa}$ determined: } \\
\cline { 4 - 7 } & Experimentally & Numerically & \multicolumn{2}{|c|}{ Experimentally } & \multicolumn{2}{c|}{ Numerically } \\
\cline { 4 - 7 } & & $\sigma_{1}$ & $\sigma_{2}$ & $\sigma_{1}$ & $\sigma_{2}$ \\
\hline 6.0 & 1.49 & 1.51 & 2.12 & 0.59 & 2.11 & 0.61 \\
\hline 14.1 & 1.12 & 1.12 & 1.11 & 0.00 & 1.12 & 0.00 \\
\hline 21.6 & 1.39 & 1.41 & 1.38 & 0.00 & 1.41 & 0.00 \\
\hline 25.0 & 1.61 & 1.60 & 1.63 & 0.00 & 1.62 & 0.00 \\
\hline 29.1 & 1.80 & 1.81 & 1.84 & 0.00 & 1.83 & 0.00 \\
\hline 37.2 & 1.68 & 1.72 & 1.70 & 0.00 & 1.72 & 0.00 \\
\hline 45.1 & 1.58 & 1.59 & 1.61 & 0.00 & 1.62 & 0.00 \\
\hline 52.8 & 1.52 & 1.51 & 1.49 & 0.00 & 1.51 & 0.00 \\
\hline 60.5 & 1.02 & 1.11 & 1.14 & 0.00 & 1.12 & 0.00 \\
\hline 62.3 & 0.89 & 0.92 & 0.89 & 0.00 & 0.92 & 0.00 \\
\hline 67.9 & 0.50 & 0.51 & 0.49 & 0.00 & 0.51 & 0.00 \\
\hline 75.0 & 1.42 & 1.41 & 0.00 & -1.42 & 0.00 & -1.40 \\
\hline
\end{tabular}


For the comparison of differences of principal normal stress $\sigma_{1}-\sigma_{2}$ and their components $\sigma_{1}, \sigma_{2}$ in the analyzed points obtained by experimental means of reflection photoelasticity the analyzed sample rotors were modeled in SolidWorks program, and stress values were calculated with the use of final element method.

\section{Summary}

Experimental analysis enabled us to identify differences of principal normal stresses $\sigma_{1}-\sigma_{2}$ in selected points in the direction of axis $x$. Listed in Tab. 1 and are coordinates of these points as well as resulting values of principal stresses $\sigma_{\text {red }}$ in the sample rotor. Values of these stresses were determined in two different ways: experimentally my means of reflection photoelasticity and numerically with the use of finite element method [12, 13].

Principal normal stresses were separated from the measured values in „Photoelast“ software [10]. Listed in Tab. 1 are values of separated principal normal stresses $\sigma_{1}$ and $\sigma_{2}$ which were determined numerically and with the use of „Photoelast“ software.

Diagrams (see Fig. 6 to 8) which are always placed after the relevant table (Tab. 1), were drawn for the prediction of normal stresses in any point on the axis $x$ (listed in given tables). Graphical representation of the above-stated relations was created by interpolation with the use of cubic splines in MATLAB environment [14].

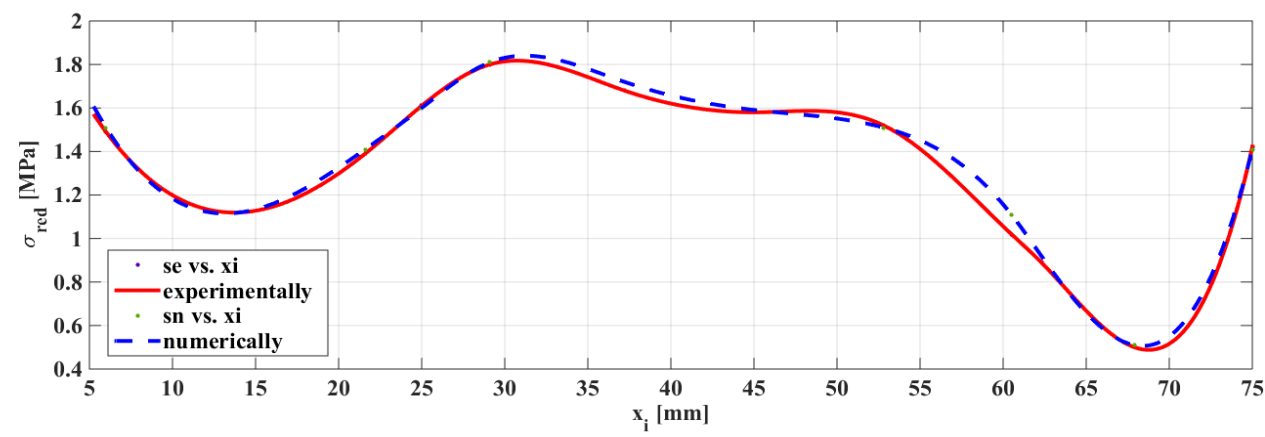

Fig. 6 Behavior of stress differences $\sigma_{\text {red }}=\sigma_{1}-\sigma_{2}$ on the sample rotor

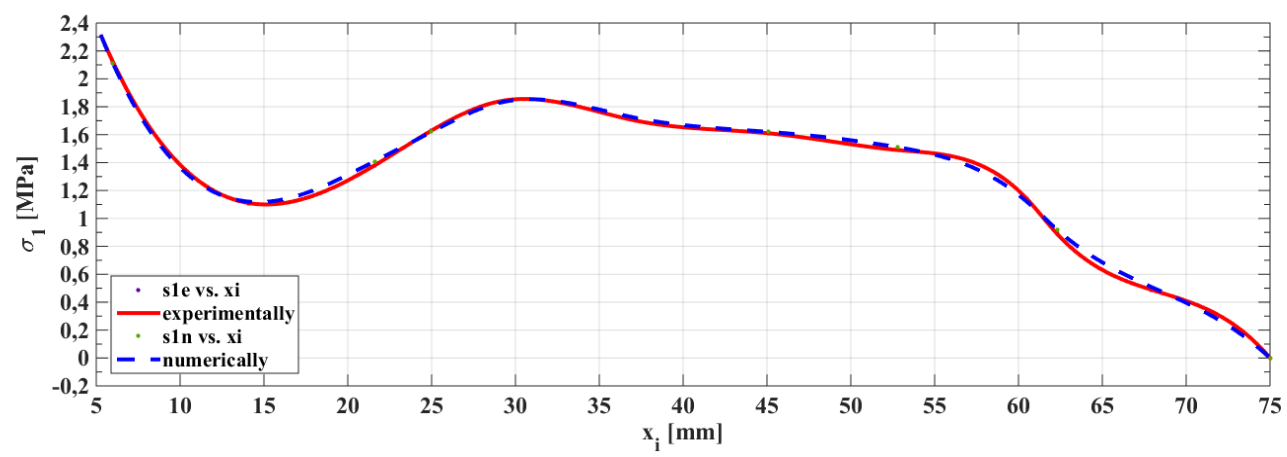

Fig. 7 Behavior of separated values of principal normal stresses $\sigma_{1}$ on the sample rotor

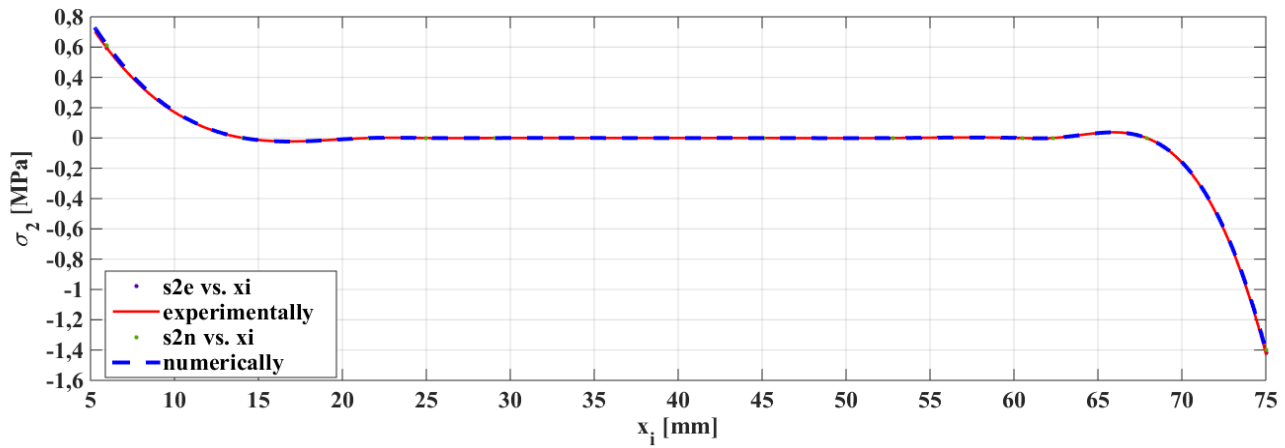

Fig. 8 Behavior of separated values of principal normal stresses $\sigma_{1}$ on the sample rotor 
Based on the observation of individual values in given tables and the comparison of diagrams it can be concluded as follows:

- There is a minimum difference between stresses which were determined experimentally and stresses which were determined numerically by means of the finite element method.

- A slightly bigger difference is between stresses which were determined stress which were determined experimentally as well as numerically. We assume that such difference might have been caused due to rectangular shape of the segment.

These deviations between stresses which were determined with the use of three methods comprise $5 \%$, which represents sufficient accuracy for practical application of reflection photoelasticity.

\section{CONCLUSION}

The presented findings certified that one of the methods based on the electromagnetic wave combined with the computer support at simultaneous application of the high-quality recording medium is considered procedure enabling the reduction of time required for the analysis of experimentally determined parameters.

The contribution of the presented paper may be summarized into the following items:

- the adjusted equations (1) and (2) applied within the method of characteristics developed a precondition of the experiment process automation;

- the „Photoelast“" program was developed for the purposes of automated isolation of the stresses right from the scanned field of the isochromatic fringes;

- methodical sequence of the experiment was established;

- the notion of aberration and its impact on the experiment were subject to the analysis;

- this solution enabled us to eliminate the experimenter's subjective mistakes made at improper identification of the isochromatic fringes order.

The measured parameters were compared with parameters of the stresses determined numerically. The minimum difference of the results makes possible to consider the prepared method and the „Photoelast“" program as relevant for its application within the photoelasticity.

\section{Acknowledgment}

This work was supported in part by the Ministry of Education of the Slovakia Foundation under grant project VEGA No. 1/0290/18 and grant project VEGA No. 1/0731/16.

\section{REFERENCES}

[1] O. Ostertag, E. Ostertagová, P. Frankovský. Aberration Problem within the Process of Automation of the Photoelastic Measurement of the Stresses. Key Engineering Materials 2014 (635), $51-56$.

[2] AS Kobayashi, editor. Handbook on experimental mechanics, 2nd revised ed. New York: VCH Publishers Inc.; 1993.

[3] P. Macura, F. Fojtík. The Application of Photostress Method for Experimental Stress Analysis of Plate Transducer. 49th International Scientific Conference, 2011, p. 195.

[4] O. Ostertag, E. Ostertagová, P. Frankovský. Photoelast Method and Possible Applications of Mathematical Statistics in Prediction of Stress State of Structural Elements. Applied Mechanics and Materials Trans Tech Publications 2014 (611), 405 - 411.

[5] B. D. Agarwal. An approximate method of orthotropic photoelastic analysis. Experimental Mechanics 1983 (23), No. 1, 55 - 58. 
[6] F. Trebuňa, F. Šimčák, P. Frankovský, R. Huňady, M. Pástor. Využitie optických metód v experimentálnej mechanike 1. Košice: TU, 2014.

[7] F. Fojtík, P. Ferfecki, Z. Paška. Computer aided evaluation of the stress tensor in the twodimensional photoelasticity. Appl. Mech. Mater. 2016 (827), 181 - 184.

[8] N. A. Dombrachev, I. A. Korshunov, A. B. Yakimovich. Based on complexity theory the automated normalization system of tooling equipment. Avtomatizatsiya $i$ Sovremennye Tekhnologii, Izdatel'stvo Mashinostroenie 2004 (10), 3 - 8.

[9] O. Ostertag, P. Frankovský, E. Ostertagová, F. Trebuňa. Application of the harmonic star method in photoelastic separation of principal stresses. Applied optics, 2016 (55), No. 3, $425-431$.

[10] Z. Paška, F. Fojtík, P. Ferfecki. Full Field Evaluation of the Stress Tensor Components in 2D Photoelasticity Via Computer Software. Journal of Mechanical Engineering Strojnícky časopis 2016 (66), No. 2, 63 - 66.

[11] R. Owsiński, A. Niesłony. Identification of Fatigue Cracks on the Basis of Measurable Changes in System Dynamics. Journal of Mechanical Engineering - Strojnícky časopis 2017 (67), No. 2, $77-84$.

[12] E. Tertel, P. Kurylo, W. Papacz. The stress state in the threelayer open conical shell during of stability loss. Acta Mechanica Slovaca 2014 (18), No. 2, 56 - 63.

[13] P. Frankovský, O. Ostertag, E. Ostertagová, F. Trebuňa, J. Kostka, M. Výrostek. Experimental analysis of stress fields of rotating structural elements by means of reflection photoelasticity. Applied Optics 2017 (56), No. 11, 3064 - 3070.

[14] E. Ostertagová, Aplikovaná štatistika v počítačovom prostredí MATLABu. Technická univerzita v Košiciach. 2015. 